\title{
Maternal Opinions on Breastfeeding, Introducing Solids, and Postpartum Weight Loss
}

Jennifer Cauble

University of Kansas Medical Center

Amy Herman

University of Kansas Medical Center

Christine M Daley

University of Kansas Medical Center

Holly R Hull ( $\square$ hhull@kumc.edu )

University of Kansas Medical Center

\section{Research}

Keywords: Maternal opinions, weight loss, breastfeeding, Early cessation of breastfeeding

Posted Date: February 19th, 2021

DOI: https://doi.org/10.21203/rs.3.rs-223745/v1

License: (c) (1) This work is licensed under a Creative Commons Attribution 4.0 International License. Read Full License 


\section{Abstract}

Objectives: Early cessation of breastfeeding, inappropriate introduction of solids, and postpartum weight retention are associated with negative health outcomes for mother and infant. The purpose of this study was to understand timing of and factors that influence infant feeding decisions and barriers that women face when breastfeeding, introducing solids, and losing weight postpartum to inform future interventions.

Methods: Eleven women who had recently delivered (infant age 3-10 months) were recruited from a Kansas City Metro pediatric office. All women were currently breastfeeding or had previous breastfeeding experience. Women completed a feeding survey and participated in a group interview to obtain information regarding breastfeeding, introducing solids, and postpartum weight loss. A content and text analysis were performed to summarize the data.

Results: Predominant emerging themes were: (1) Inadequate knowledge for caring for self and infant (2) Desired knowledge from a Healthcare Provider (3) Feeding decisions were made before pregnancy and preparation took place in the second/third trimesters (4) Unmet breastfeeding goals (5) Clear guidelines on introducing solids are lacking (6) ) Those that achieve pre-pregnancy weight have a different body shape and weight distribution (7) and Lack of time and energy make postpartum weight loss hard.

Conclusions: Overall, women do not feel they have adequate information to successfully breastfeed, introduce solids, or lose weight postpartum. They would like basic and practical information on both caring for and feeding themselves and their infant. They would like this information provided to them both during pregnancy and during well child visits.

\section{Background}

The transition to motherhood can be challenging. Infant feeding and postpartum weight loss are two areas that can be difficult to navigate. Breastfeeding is the recommended standard for infant feeding and provides mother and baby many health related benefits[1]. Infants who are breastfed have a decreased risk of infant infection [2, 3], childhood obesity [4], diabetes [5], childhood cancer [6], and also a 50\% reduction in the risk of sudden infant death syndrome [7]. Maternal benefits include a reduction in maternal blood loss [8], ovarian [9] and breast cancer risk [10], postpartum depression [11], pregnancy related weight retention [12, 13], and encourages child-spacing [14].

Exclusive breastfeeding is recommended by the American Academy of Pediatrics (APP) for the first six months with continued breastfeeding to 12 months $[1,15,16]$. At about six months, solid foods can be introduced. In the United States (US), only $24.9 \%$ of babies are exclusively breastfed at six months [17] and $40.4 \%$ of infants are given solids before the recommended age [18]. Early introduction of solids is associated with a number of medical issues such as eczema [19], celiac disease [20], and diabetes [21, 22]. There is also an associated increased obesity risk with early introduction of solids [4, 23-25]. Weight retained from pregnancy can lead to increased rates of maternal obesity at one year postpartum [26] and is linked to poor health outcomes such as diabetes, cardiovascular disease, and depression [27]. 
Furthermore, maternal weight retention increases the chance of entering a subsequent pregnancy overweight or obese, thus predisposing the infant to obesity, and perpetuating obesity generation to generation.

Interventions to improve breastfeeding rates $[28,29]$ and postpartum weight loss $[30]$ provide mixed results and interventions to improve the timing of introducing solids are lacking. Overall, little is known about when women make their infant feeding decisions, what information is desired, or how and when they would like the information delivered to improve success rates. To our knowledge, no studies have addressed how mothers feel about introducing solids to their infant for the first time. Furthermore, additional work needs to be done to understand what information women need to achieve weight loss in the postpartum period. This knowledge is important to inform effective intervention development. Therefore, the purpose of this study was to gain knowledge on when women make their infant feeding decisions, what factors influence infant feeding decisions, and gain understanding from experienced women regarding barriers surrounding successful breastfeeding, introduction of solids, and postpartum weight loss to help inform future interventions.

\section{Methods}

\section{Study Aim and Design}

This project was completed to understand maternal attitudes and beliefs surrounding breastfeeding, introduction of solids, and postpartum weight loss to help inform future interventions. Specifically, the study was developed to;

1. Identify barriers faced during breastfeeding, introduction of solids, and postpartum weight loss.

2. Identify what information or support would be helpful regarding breastfeeding, introduction of solids, and postpartum weight loss.

3. Identify at what stage of pregnancy would be best to receive information on breastfeeding, introduction of solids, and postpartum weight loss.

Both qualitative and quantitative methods were used for this project. The primary data collection and analyses were qualitative, using group interviews to understand barriers, when women need additional support or education, and what information would be beneficial. Quantitative data collection to supplement the group interviews included questionnaires to obtain demographic information and infant feeding practices. The group interview data were also analyzed quantitatively with a content analysis to summarize the data.

\section{Sampling Frame}

Four group interviews were conducted with a purposive sample of 11 women who had recently delivered (infant age 3-10 months) and had previous breastfeeding experience. While purposive sampling is a nonprobability technique, it was appropriate for this study because only women who had previously 
delivered an infant with breastfeeding experience would be able to identify specific needs and barriers to infant feeding and postpartum weight loss. One scheduled group had only one participant attend; an interview was conducted using the group interview questions. Informed consent was obtained in a private exam room at the pediatric office, either at a regular pediatric appointment or before the group interview. Demographic and infant feeding surveys were administered after participants provided consent and prior to the start of the group interviews.

\section{Recruitment}

Study protocols were approved by the University of Kansas Medical Center's Human Subjects Committee (HSC \# STUDY00003055). Participants were recruited from a Kansas City metro pediatric office. Flyers were put up around the clinic and women involved in a weekly breastfeeding support group run by the clinic were approached by the support group leader.

\section{Data Collection}

\section{Group Interviews}

Group interviews were held at the pediatric office where recruitment took place. Sessions were in the evenings to accommodate women's work schedules. The moderator's guide was developed by the principal investigator ( $\mathrm{Pl} ; \mathrm{HH}$ ) and the research coordinator (JSC), who is a lactation consultant. Structured questions were asked about barriers, knowledge, and experiences related to infant feeding and postpartum weight loss (see Table 1 for questions). The same research coordinator moderated all group interviews. Group sessions lasted 30-60 minutes and were recorded and transcribed verbatim. The study was not designed to reach saturation, but to provide a needs assessment to inform intervention development. Once transcripts were produced, a research assistant (AH) reviewed all transcriptions for accuracy. Any discrepancies were modified as necessary by the research coordinator (JC) until agreement was reached. 
Table 1

Group Interview Questions

1. "At what point did you decide you were going to breastfeed your child?"

a. Did this time differ if this was not your first child?

2. "Prior to delivery what was your plan on how long you wanted to breastfeed your child?"

a. Did you meet your goals?

b. If you did not meet your goals, what do you think affected this?

3. "What was the length of time that you breastfed your infant and what barriers did you face?"

4. "At what time would you have liked information on breastfeeding?"

- During pregnancy? (If so, when?)

- After the baby was born? (If so, when?)

5. "What were the most difficult points in time regarding breastfeeding and how long did it take before you felt you had things under control?"

6. "If formula was introduced at any time, at what time was it introduced and what was your reason for introduction?"

7. "What were your plans for introducing solids foods prior to delivery and how did you determine this plan?"

8. "If you did not introduce solids as planned, when did you introduce solids and why did your plan change?"

9. "What barriers did you encounter while attempting to get back to pre-pregnancy weight?"

10. "Were you able to achieve pre-pregnancy weight and if so at what time point postpartum did you reach it?

11. "If you had access to a weekly support group via phone led by a Registered Dietitian/Lactation Consultant, with information and support regarding breastfeeding, postpartum weight loss, and introduction of solid foods would you use this support group?"

\section{Questionnaires}

Women were given questionnaires to collect demographic information and infant feeding practices. The demographic questionnaire collected data including pre-pregnancy body mass index (BMI) based on selfreported height and pre-pregnancy weight, age, sociodemographics (income, education, and employment status), number of prior pregnancies, and whether they were currently or recently pregnant. The feeding questionnaire collected data regarding breastfeeding plan and initiation, breastfeeding goals, formula introduction, age of solid food introduction, and amount of postpartum weight loss.

\section{Data Analysis}




\section{Group Interviews}

Content Analysis: Verbatim transcripts were coded by hand by the research coordinator and topics were identified within each question to create a key for analysis. Final transcripts and the analysis key were given to three individual study personnel (the research coordinator; JSC, research assistant; AH, and PI; $\mathrm{HH}$ ), who deductively abstracted the data into topics. The study team then met and reviewed each question individually. Frequencies were determined to summarize the data.

Text analysis: Each of the three members of the research team deductively coded the transcripts. All coders identified preliminary themes which were sent to an outside researcher to develop thematic statements. The outside researcher and the study team then met to check and discuss final themes. All illustrative quotes were identified by the moderator/research coordinator.

\section{Questionnaire}

Using data from the demographic questionnaire, proportions were calculated to describe the population. Rates and percentages calculated from the infant feeding questionnaire provided information on quantifiable goals and practices of the women regarding breastfeeding, introducing solids, and postpartum weight loss. These data allowed us to determine if women met national recommendations for feeding practices and was used to supplement the group interview data.

\section{Results}

Four group interviews were held yielding eleven women ranging in age from 21-43 years (mean $32.0 \pm$ 6.9) with current or previous breastfeeding experience participated. All women had recently delivered and had infants between the age of three and 10 months. Most women already had children, with the following sample distribution; one child (27.3\%), two children (36.4\%), three children (18.2\%). All women had at least a high school diploma with $63.7 \%$ of women having an undergraduate or postgraduate degree, $45.5 \%$ had full-time or part-time employment, and all women were white and non-Hispanic. Participant demographics are displayed in Table 2. Women reported the following plans for breastfeeding. Ten women planned to breastfeed prior to delivery and all 10 women initiated breastfeeding in the hospital. Over $70 \%$ of women planned to breastfeed for at least one year, however, $30 \%$ of women only breastfed for $0-3$ months, $20 \%$ breastfed for $9-12$ months, and $40 \%$ were still breastfeeding at the time of the group interview (age nine months). Sixty percent of women introduced formula at some point. Forty percent of women did not feel they met the breastfeeding goal they set for themselves. Regarding introducing solids, $22 \%$ of women stared at four months, $11 \%$ at five months, and $67 \%$ at six months. Forty percent of women stated they achieved pre-pregnancy weight. One woman achieved pre-pregnancy weight before three months, one at four months, one at six months, and one after six months. 
Table 2

Maternal Characteristics

\begin{tabular}{|ll|}
\hline Demographics $(\mathbf{n}=\mathbf{1 1})$ & $32.0, \pm 6.9$ \\
\hline Age (in years, mean, SD) & \\
\hline Race & $100.0 \%$ \\
\hline White & \\
\hline Ethnicity & $100.0 \%$ \\
\hline Non-Hispanic/Latino & \\
\hline Education Level & $27.3 \%$ \\
\hline High School Diploma & $9.1 \%$ \\
\hline Associates Degree & $36.4 \%$ \\
\hline Undergraduate Degree & $27.3 \%$ \\
\hline Postgraduate Degree (masters, PhD) & \\
\hline Employment Status & $45.5 \%$ \\
\hline Stay at home to watch children & $27.3 \%$ \\
\hline Part-time & $27.3 \%$ \\
\hline Full-time & \\
\hline Number of Pregnancies & $18.2 \%$ \\
\hline 1 & $27.3 \%$ \\
\hline 2 & $36.4 \%$ \\
\hline 3 & $18.2 \%$ \\
\hline 4 & \\
\hline
\end{tabular}

Thematic analysis of group interview data revealed seven overarching themes: (1) inadequate knowledge for caring for self and infant (2) women desired knowledge from a Healthcare Provider (3) feeding decisions were made before pregnancy and preparation took place in the second/third trimesters (4) breastfeeding goals were not met (5) clear guidelines for introducing solids were lacking (6) in those achieving pre-pregnancy weight, their body shape and weight distribution changed (7) and a lack of time and energy make postpartum weight loss hard. Exemplary quotes are described in Table 3. 


\section{Cross Cutting Statements}

1. Women do not have adequate knowledge on feeding and caring for their infants or themselves. Women want practical information, both during pregnancy and during standard well child visits, on how to be a mother, caring for a feeding a new infant and caring for themselves.

2. Women actively seek information from family, friends, internet, and books but would like the information to be obtained from their healthcare provider.

\section{Breastfeeding Statements}

3. Women make decisions about infant feeding prior to pregnancy and desire information about breastfeeding during the $2^{\text {nd }}$ or $3^{\text {rd }}$ trimester

"I thought before I was pregnant that I knew how I wanted to feed my baby."

"The second trimester... In the first trimester I was too busy worried about ya know, getting sick all the time and trying to survive the day and then third trimester I was, you know, laying in bed."

4. Women set goals to breastfeed for 1 year or longer but do not reach these goals due to the primary barriers of milk supply and latching issues

"My goal was to nurse for a year, and no, I did not meet my goal. Like not even close. It was because of milk supply, just not having enough."

\section{Introducing Solids Statements}

5. Women want clear guidelines on when and how to introduce solids to their infants

"I think there is too much information out there and I kind of get tired of all the people's opinions."

\section{Postpartum Weight Loss Statements}

6. Some women achieve pre-pregnancy weight but do not feel their bodies are the same. Women are unprepared for this.

"I am back to pre-pregnancy weight, but my clothes still don't fit right. It's the worst."

"Weight wise I was pre-pregnancy, but my clothes no longer fit. That was a shock."

7. Women face many barriers related to postpartum weight loss but the most important are time and/or energy

"The biggest barriers are time and energy and the desire to do it, and sometimes the resources of, you know, finding things that you want to do with groups and whatever."

Theme 1 and 2: Inadequate Knowledge for Caring for Self and Infant; Desired knowledge from a Healthcare Provider

Women do not feel knowledgeable on how to feed their infant or how to generally care for their babies and themselves. They indicated that they wanted resources to help become educated on basic 
information regarding being a mother, caring for and feeding a new infant, and caring for themselves in the postpartum period. Women wanted this information during pregnancy in addition to receiving education at standard pediatric or postpartum visits. Breastfeeding education was preferable during pregnancy, but they also appreciated anticipatory guidance on introducing solids, maternal health, and postpartum weight loss. Mothers felt introducing solids should be thoroughly reviewed at a standard four-month pediatric visit. Specific information included feeding problems such as constipation or picky eating, appropriate weight gain and growth expectations, allergies, and baby led weaning. Regarding postpartum weight loss, women wanted information on realistic weight loss expectations and the effect of weight loss on milk supply. Women were already seeking out the desired information from a variety of sources including family, friends, the internet, books, etc. However, they preferred this information come from a trusted healthcare professional. Women did not agree on how they would like to receive this information, but several options were discussed including verbal information, printed information, or via technology such as phone, internet, or email.

Breastfeeding Theme 3 and 4: Feeding decisions before pregnancy and preparation in the second/third trimesters; Unmet breastfeeding goals

All 11 participants indicated that they made their feeding decision prior to pregnancy. Ten of the 11 women who participated in the study planned to breastfeed. When asked about this decision, a comment from one mother on the topic was "It's a funny question because I think it was just always known you should breastfeed your baby. My mom had talked about it, and other friends had done it. You just heard in your head that breastfeeding is best. I liked the idea of bonding and just wanted that relationship with my baby so breastfeeding was on my mind." Seventy percent of women set breastfeeding goals for up to one year or longer. Despite many women having goals that aligned with breastfeeding recommendations, $40 \%$ of the women did not meet their goal for breastfeeding length. One mom indicated that "My plan was to nurse her for a year and I did not meet my goal, and I was not even close. The reason was because I did not have enough milk, and I supplemented her with formula until she was done nursing and then went to formula only." Milk supply issues was a top stated barrier for not meeting breastfeeding goals. In addition to milk supply issues, women named several other barriers including latching issues $(n=3)$, maternal health $(n=2)$, nipple pain $(n=1)$, and other $(n=2)$. "Other" reasons included mother being pregnant and an infant milk allergy. To address these barriers, women wanted basic information on breastfeeding, difficulties experienced while breastfeeding and how to handle problems that arise, and where they can find support. Specific breastfeeding information desired included proper use of a nipple shield, guidance for moms who are exclusively pumping, how often to feed their baby, hunger cues versus other reasons the baby may cry, latching techniques, and how to access a lactation consultant. Women also indicated that it is important for mothers to have an understanding that facing barriers is normal and it does not make them a bad mother. One mom stated that "I think it is important for new mothers who have never breastfed to understand it is not easy because it is one of the most difficult things I have ever done, and you really have to be persistent at it."

Introducing Solids Theme 5: Clear recommendations on introducing solids 
The primary message from women regarding introducing solids was that they wanted clear guidelines on when and how to introduce solids. In general, women planned to introduce solids around six months of age. Seventy-two percent of women indicated that a healthcare provider or expert committee recommendations influenced their decision on when to start solids. Despite this, women sought out information on introducing solids from a source other than their provider such as a technological source (i.e., internet, video, etc.), book/article, or friend. When asked if they would utilize a specific manual provided from their doctor, all women indicated that they would like and utilize this. The standard fourmonth pediatric visit was the favorable time to receive this resource. When asked what barriers the women faced when introducing solids, the most common response was being overwhelmed with information or that their child was not interested in food in general; however, they also cited barriers such as medical problems like eczema and allergies or difficulty getting the child to sit still for a meal. One mom said "I think there is too much information out there and I get tired of other people's opinions. So I selected my doctor carefully so I can put my trust in him and I won't have to worry about every little thing I read and every little trend because it's too overwhelming." Another mom said "I am frustrated because of his total disregard for food. He will put anything in his mouth besides food. So that was a struggle." Women indicated wanting practical information for how often to feed their baby and how much to offer, recipes for making baby food, information on picky eating, how to combat constipation, guidelines related to food allergies, and information about appropriate growth and weight gain.

Postpartum Weight Loss Theme 6 and 7: Those that achieve pre-pregnancy weight have a different body shape and weight distribution; Lack of time and energy make postpartum weight loss hard

Many women in this study were able to achieve pre-pregnancy weight, but three women specifically mentioned that their bodies were not the same. Women stated this was not an expectation prior to pregnancy. One woman said "Weight wise I was back to my pre-pregnancy weight, but my clothes no longer fit. That was a shock." Another said "I am back to pre-pregnancy weight, but my clothes still don't fit right. It's the worst." The women indicated that they wanted to be informed of realistic expectations regarding postpartum weight loss as well as the fact that their bodies may not be the same despite achieving pre-pregnancy weight. Women named several barriers related to postpartum weight loss including hunger related to breastfeeding, milk supply concerns, a lack of ability to exercise, and turning to convenience junk food. However, the most consistent barriers were lack of time and/or energy. One mom stated "The biggest barriers are time and energy and the desire to do it, and sometimes the resources of finding things that you want to do with groups." Another said "Time for exercise is a barrier. I just can't seem to fit it in." Women indicated that they would like support to help overcome these barriers. Examples of information women wanted included time management strategies, support systems, childcare options during exercise, available exercise programs, and practical information on diet and nutrition. Many women commented on being interested in some type of postpartum group and felt that having appropriate support or a community of other mothers would improve their postpartum weight loss experience. 


\section{Discussion}

The primary goal of this study was to gain an understanding of when women make their infant feeding decisions, what factors influence those decisions, and understand what barriers women face when breastfeeding, introducing solids, and returning to pre-pregnancy weight to inform future interventions. Effective interventions need to be tailored to the targeted population to improve breastfeeding rates, introduction of solids, and postpartum weight loss. This study found women feel inadequately educated on these areas. Women desire the education; however, the education is not being provided by the healthcare professionals at the appropriate time. The lack of education and support hinders the ability to meet personal or recommended breastfeeding goals, feel confident to introduce solids appropriately, and have the resources and tools to overcome barriers to facilitate postpartum weight loss. Women want to be educated by a healthcare professional on infant feeding and caring for themselves while they are still pregnant and they want additional resources after the baby has arrived to complement the knowledge they have.

\section{Breastfeeding}

To our knowledge, this is the first study to report on when women are making infant feeding decisions. Study participants unanimously indicated they decided before becoming pregnant if they were going to breastfeed or formula feed. Only one woman planned to formula feed and this was due to medical complications and a negative previous breastfeeding experience. This finding may suggest public health messages or societal norms related to breastfeeding have an impact on a women's decisions before she becomes pregnant. Therefore, interventions to target improved breastfeeding rates may need to occur preconception. Further research should investigate this finding further as there is the potential that outcomes would be different based on maternal age, parity, and status of a planned or unplanned pregnancy.

Current interventions to improve breastfeeding rates are primarily delivered during the postpartum period [28]. We found that women make their infant feeding decision prior to pregnancy but would like educational information during the prenatal period. Data suggest that prenatal interventions improve breastfeeding rates. A recent study by Schreck et al. compared a control group to an intervention that combined one on one prenatal education by an International Board Certified Lactation Consultant (IBCLC) combined with a free breastfeeding support group in the postpartum period. When compared to the control group, women who received the intervention had higher breastfeeding initiation rates, but breastfeeding duration rates did not differ. However, women who received the intervention and attended postnatal breastfeeding support groups had both higher breastfeeding initiation rates and prolonged breastfeeding [31]. This suggests that the educational component is beneficial for both initiation and early breastfeeding struggles but additional hands on support (that is typically offered at a breastfeeding support group) may be required for continued breastfeeding or addressing struggles that occur further into the postpartum period.

Women often encounter a number of breastfeeding struggles that lead to early cessation including sore nipples, inadequate milk supply, infant problems, and the perception that the infant is not satiated [32]. 
Similarly, the primary issues women faced in this study were latching issues and milk supply issues [33]. Our study expanded on the barriers and investigated the information that would be beneficial to improve these struggles. Some struggles may require a different mode of delivery for the information. For example, teaching women about appropriate milk supply and breastfeeding basics can occur prenatally via educational material or classes; however, teaching appropriate latching techniques may need to be taught in person. Some women indicated that learning theoretical information about proper latching technique is simply not enough, they need hands on support once their baby has arrived. In summary, women want to be educated during pregnancy in preparation for breastfeeding but feel they may also need additional hands on support if/when struggles arise.

\section{Introducing Solids}

The current recommendation for introduction of solids is about six months in conjunction with breastfeeding or formula feeding [1]. However, according to the Infant Feeding Practices Study II data, about $40 \%$ of infants are given solids prior to four months of age [18]. This is concerning due to the associated medical complications related to early solid introduction [19-25]. We found no women started solids prior to four months of age. Even though they followed the current recommendation for timing of solids introduction, women indicated feeling unprepared for the transition and cited barriers of feeling overwhelmed by information from a combination of sources such as family, friends, and online baby forums, with no clear guidance from their healthcare providers. This is not surprising given there is disagreement between expert committees on when solids should be introduced. The AAP Committee on Nutrition recommends the introduction of solid foods between four and six months of age, with developmentally readiness as a guide [34], while the AAP Section on Breastfeeding recommends exclusive breastfeeding until "about" six months [1]. In addition, neither the AAP Committee on Nutrition nor the AAP Section on Breastfeeding offer clear guidelines on what foods to start with or the frequency and amount of food to provide. These conflicting recommendations cause inappropriate introduction of solids due to confusion or misunderstanding of the guidelines and contribute to a lack of maternal confidence in the ability to properly feed her child.

Interventions aimed at the appropriate introduction of solid foods are lacking. A recent review by Arikpo et al. found educational interventions decrease the risk for early introduction of solids, but they are limited and are occurring primarily in the postpartum period [29]. The lack of interventions aimed at improving both the timing of introduction of solids and maternal self-efficacy presents a novel opportunity to develop an effective intervention to both improve health outcomes and add to the scientific body of knowledge. Our study provides the specific informational framework for what should be included in such an educational intervention.

\section{Postpartum Weight Loss}

Postpartum weight loss is difficult. Thirteen to twenty percent of women retain five $\mathrm{kg}$ or more at 12 months [35]. This is concerning as it may progress a women into a higher BMI category and expose her to negative health outcomes such as gestational diabetes with a subsequent pregnancy [36], type 2 
diabetes later in life [37], cardiovascular disease, and depression [27]. Forty percent of participants reported returning to their pre-pregnancy body weight. Even though they returned to their pre-pregnancy body weight, it was clear many felt their body weight was redistributed to different locations. Further, participants indicated they wanted to exercise or that they understood exercise would aid in postpartum weight loss. Despite this, participants stated they could not find the time or did not have the desire to exercise. Other studies have found similar results. Ostbye et al. [38, 39] randomized women to 10 group physical activity sessions, eight healthy eating classes, and six phone counseling sessions or to a control group over a nine-month period. No between group difference was found for weight change at 12 months postpartum. Authors cited reasons for the null results were low intervention participation due to time barriers. The authors suggested a home-based intervention (via mail, email, telephone, internet, etc) would be a more viable option for new mothers as it would address barriers such as time and childcare. Authors also discussed offering the intervention in the prenatal period to address time and childcare barriers; however, it is unknown if behaviors learned prenatally would be implemented postpartum. To our knowledge, no studies have investigated prenatal interventions to address postpartum weight loss. Overall, women want information to help them lose weight in the postpartum period and are receptive to prenatal education regarding postpartum weight loss. They desire practical information on time management, healthy eating, maintaining a milk supply while losing weight, and tips for exercising with baby.

\section{Limitations}

Our study is limited by the small sample size and lack of diversity in our participants. The study participants were older and had multiple children. First time mothers may require or desire different information for success when compared to women with multiple children. Further, a first-time mother may be more interested in gaining new knowledge and have more time to participate in a pregnancy intervention when compared to women who already have children. Additionally, more tech savvy women may prefer to receive information in different ways (ie. technology based versus in person). Women in our study did not introduce solids early but nationwide $40.4 \%$ of infant are given solids too early [18]. It would be important to understand what influences early introduction of solids. This knowledge would complement the results of our study. While we feel our findings of what women want to be successful are representative, additional research with a larger sample composed of a larger variety of women would be beneficial.

\section{Conclusions}

Overall, women would like more information to manage their own health and the health of their infant. Women do not feel they have adequate information or support to reach their goals. Educational interventions during pregnancy could help fill this knowledge gap. Interventions should provide practical tips and basic information on how to be a mother and care for an infant, basics of breastfeeding, clear cut guidelines on when and how to introduce solids, and information on healthy eating, exercise, and time management to aid in postpartum weight loss. These tips and resources should be developed by a 
healthcare provider and delivered by a knowledgeable professional in breastfeeding, introducing solids, and postpartum weight loss. There are several ways that this information could be delivered, but it should be tailored to the specific population.

\section{Abbreviations}

AAP: American Academy of Pediatrics; PI: Principal Investigator; BMI: Body Mass Index; IBCLC: International Board Certified Lactation Consultant

\section{Declarations}

\section{Ethics approval and consent:}

Data reported was collected under the protocols approved by the Institutional Review Board (\#STUDY00003055) at the University of Kansas Medical Center. All participants gave written informed consent prior to data collection.

\section{Consent for publication:}

Not applicable

\section{Availability of data and materials:}

The data will not be available for public deposition.

\section{Competing interest:}

Each author has declared no conflict of financial or personal interests in any company or organization sponsoring this study.

\section{Funding:}

Not applicable

\section{Authors' contributions:}

$\mathrm{JC}$ conceived the study idea, participated in data collection and analysis, manuscript writing and editing. AH participated in data analysis, manuscript writing and editing. CD participated in data analysis and manuscript editing. $\mathrm{HH}$ participated in study conception, provided study supervision, participated in data analysis and manuscript editing. All authors read and approved the final manuscript.

\section{Acknowledgements:}

The authors would like to acknowledge Dr. Scott Rogers, DO, the staff at Priority Care Pediatrics, and the study participants for their gracious support. 


\section{References}

1. Section on, B., Breastfeeding and the use of human milk. Pediatrics, 2012. 129(3): p. e827-41.

2. Howie, P.W., et al., Protective effect of breast feeding against infection. Bmj, 1990. 300(6716): p. 11-6.

3. Beaudry, M., R. Dufour, and S. Marcoux, Relation between infant feeding and infections during the first six months of life. J Pediatr, 1995. 126(2): p. 191-7.

4. Kramer, M.S., Do breast-feeding and delayed introduction of solid foods protect against subsequent obesity? J Pediatr, 1981. 98(6): p. 883-7.

5. Owen, C.G., et al., Does breastfeeding influence risk of type 2 diabetes in later life? A quantitative analysis of published evidence. Am J Clin Nutr, 2006. 84(5): p. 1043-54.

6. Davis, M.K., D.A. Savitz, and B.I. Graubard, Infant feeding and childhood cancer. Lancet, 1988. 2(8607): p. 365-8.

7. Vennemann, M.M., et al., Does breastfeeding reduce the risk of sudden infant death syndrome? Pediatrics, 2009. 123(3): p. e406-10.

8. Sobhy, S.I. and N.A. Mohame, The effect of early initiation of breast feeding on the amount of vaginal blood loss during the fourth stage of labor. J Egypt Public Health Assoc, 2004. 79(1-2): p. 112.

9. Feng, L.P., H.L. Chen, and M.Y. Shen, Breastfeeding and the risk of ovarian cancer: a meta-analysis. J Midwifery Womens Health, 2014. 59(4): p. 428-37.

10. Zhou, Y., et al., Association Between Breastfeeding and Breast Cancer Risk: Evidence from a Metaanalysis. Breastfeed Med, 2015. 10: p. 175-82.

11. Borra, C., M. lacovou, and A. Sevilla, New evidence on breastfeeding and postpartum depression: the importance of understanding women's intentions. Matern Child Health J, 2015. 19(4): p. 897-907.

12. Newcomb, P.A., et al., Lactation and a reduced risk of premenopausal breast cancer. N Engl J Med, 1994. 330(2): p. 81-7.

13. McCoy, S.J., et al., Risk factors for postpartum depression: a retrospective investigation at 4-weeks postnatal and a review of the literature. J Am Osteopath Assoc, 2006. 106(4): p. 193-8.

14. van Unnik, G.A. and J. van Roosmalen, [Lactation-induced amenorrhea as birth control method]. Ned Tijdschr Geneeskd, 1998. 142(2): p. 60-2.

15. Sobti, J., G.P. Mathur, and A. Gupta, WHO's proposed global strategy for infant and young child feeding: a viewpoint. J Indian Med Assoc, 2002. 100(8): p. 502-4, 506.

16. Committee on Health Care for Underserved Women, A.C.o.O. and Gynecologists, ACOG Committee Opinion No. 361: Breastfeeding: maternal and infant aspects. Obstet Gynecol, 2007. 109(2 Pt 1): p. 479-80.

17. Centers for Disease, C. Breastfeeding Report Card. 2018; Available from: https://www.cdc.gov/breastfeeding/data/reportcard.htm. 
18. Clayton, H.B., et al., Prevalence and reasons for introducing infants early to solid foods: variations by milk feeding type. Pediatrics, 2013. 131(4): p. e1108-14.

19. Tarini, B.A., et al., Systematic review of the relationship between early introduction of solid foods to infants and the development of allergic disease. Arch Pediatr Adolesc Med, 2006. 160(5): p. 502-7.

20. Szajewska, H., et al., Systematic review: early infant feeding and the prevention of coeliac disease. Aliment Pharmacol Ther, 2012. 36(7): p. 607-18.

21. Agostoni, C., et al., Complementary feeding: a commentary by the ESPGHAN Committee on Nutrition. J Pediatr Gastroenterol Nutr, 2008. 46(1): p. 99-110.

22. Rosenbauer, J., et al., Early nutrition and risk of Type 1 diabetes mellitus--a nationwide case-control study in preschool children. Exp Clin Endocrinol Diabetes, 2007. 115(8): p. 502-8.

23. Huh, S.Y., et al., Timing of solid food introduction and risk of obesity in preschool-aged children. Pediatrics, 2011. 127(3): p. e544-51.

24. Gaffney, K.F., P. Kitsantas, and J. Cheema, Clinical practice guidelines for feeding behaviors and weight-for-age at 12 months: a secondary analysis of the Infant Feeding Practices Study II. Worldviews Evid Based Nurs, 2012. 9(4): p. 234-42.

25. Grote, V. and M. Theurich, Complementary feeding and obesity risk. Curr Opin Clin Nutr Metab Care, 2014. 17(3): p. 273-7.

26. Endres, L.K., et al., Postpartum weight retention risk factors and relationship to obesity at 1 year. Obstet Gynecol, 2015. 125(1): p. 144-52.

27. Chescheir, N.C., Global obesity and the effect on women's health. Obstet Gynecol, 2011. 117(5): p. 1213-22.

28. Skouteris, H., et al., Interventions designed to promote exclusive breastfeeding in high-income countries: a systematic review. Breastfeed Med, 2014. 9(3): p. 113-27.

29. Arikpo, D., et al., Educational interventions for improving primary caregiver complementary feeding practices for children aged 24 months and under. Cochrane Database Syst Rev, 2018. 5: p. CD011768.

30. Amorim, A.R., Y.M. Linne, and P.M. Lourenco, Diet or exercise, or both, for weight reduction in women after childbirth. Cochrane Database Syst Rev, 2007(3): p. CD005627.

31. Schreck, P.K., et al., Both Prenatal and Postnatal Interventions Are Needed to Improve Breastfeeding Outcomes in a Low-Income Population. Breastfeed Med, 2017. 12: p. 142-148.

32. Ahluwalia, I.B., B. Morrow, and J. Hsia, Why do women stop breastfeeding? Findings from the Pregnancy Risk Assessment and Monitoring System. Pediatrics, 2005. 116(6): p. 1408-12.

33. Dewey, K.G., M.J. Heinig, and L.A. Nommsen-Rivers, Differences in morbidity between breast-fed and formula-fed infants. J Pediatr, 1995. 126(5 Pt 1): p. 696-702.

34. American Academy of Pediatrics. Committee on Nutrition. and L.A. Barness, Pediatric nutrition handbook. 6th ed. 2009, Elk Grove Village, IL: American Academy of Pediatrics. xlix, 1470 p. 
35. Gunderson, E.P., Childbearing and obesity in women: weight before, during, and after pregnancy. Obstet Gynecol Clin North Am, 2009. 36(2): p. 317-32, ix.

36. Solomon, C.G., et al., A prospective study of pregravid determinants of gestational diabetes mellitus. Jama, 1997. 278(13): p. 1078-83.

37. Lee, A.J., et al., Gestational diabetes mellitus: clinical predictors and long-term risk of developing type 2 diabetes: a retrospective cohort study using survival analysis. Diabetes Care, 2007. 30(4): p. 87883.

38. Ostbye, T., et al., Active Mothers Postpartum (AMP): rationale, design, and baseline characteristics. J Womens Health (Larchmt), 2008. 17(10): p. 1567-75.

39. Ostbye, T., et al., Active Mothers Postpartum: a randomized controlled weight-loss intervention trial. Am J Prev Med, 2009. 37(3): p. 173-80. 